FERMILAB-Conf-94/041-E

$\mathbf{E 6 6 5}$

\title{
Exclusive Vector Meson Production in \\ Muon-Nucleus Scattering
}

\author{
Guang Yin Fang \\ For the E665 Collaboration \\ Harvard University \\ Cambridge, MA 02138 \\ Fermi National Accelerator Laboratory \\ P.O. Box 500, Batavia, Illinois 60510
}

February 1994

Presented at the XXIII International Multi-Particle Dynamics Symposium, Aspen, Colorado, September 13-17, 1993 


\section{Disclaimer}

This report was prepared as an account of work sponsored by an agency of the United States Government. Neither the United States Government nor any agency thereof, nor any of their employees, makes any warranty, express or implied, or assumes any legal liability or responsibility for the accuracy, completeness, or usefulness of any information, apparatus, product, or process disclosed, or represents that its use would not infringe privately owned rights. Reference herein to any specific commercial product, process, or service by trade name, trademark, manufacturer, or otherwise, does not necessarily constitute or imply its endorsement, recommendation, or favoring by the United States Government or any agency thereof. The views and opinions of authors expressed herein do not necessarily state or reflect those of the United States Government or any agency thereof. 


\title{
EXCLUSIVE VECTOR MESON PRODUCTION IN MUON-NUCLEUS SCATTERING
}

\author{
GUANG YIN FANG \\ Department of Physics \\ Harvard University \\ Cambridge, MA 02138, USA \\ (Representing the E665 Collaboration)
}

\begin{abstract}
Preliminary results on the cross section ratios of exclusive incoherent $\boldsymbol{\rho}^{0}$ and $\phi$ meson production off deuterium, carbon, calcium, and lead to that off hydrogen and coherent $\rho^{0}$ and $\phi$ meson production off calcium and lead to that off carbon in deep-inelastic muon-nucleon and muon-nucleus scattering are reported. The data were taken with the E665 spectrometer using the Fermilab Tevatron muon beam. The mean beam energy was $470 \mathrm{GeV}$. Increases in the cross section ratios are seen in both the elastic and quasi-elastic production as the four-momentum squared of the virtual photon increases. The results support the idea of color transparency.
\end{abstract}

Electro-production of exclusive vector mesons from complex nuclei was suggested as a way of testing the idea of a "shrinking photon" some time ago. ${ }^{1}$ More recently, it has been suggested as a way of testing the idea of color transparency..$^{2-4}$ A recent example is the calculation of the $A$ dependence of the ratio of cross sections of exclusive $\rho^{0}$ production from iron to that from hydrogen by Kopeliovich et al.. ${ }^{4}$

Color transparency is a novel property of color gauge invariance in quantum chromodynamics. It predicts the absence of final-state re-interactions in hard exclusive processes. Thus one expects the total cross section of a hard process that involves nucleons from a nucleus to be proportional to $\mathrm{A}$, the total number of nucleons in the nucleus. To experimentally observe color transparency one needs not only a process that selects out small-configuration hadrons but also high enough energy such that the emerging hadrons do not expand appreciably while propagating through the nucleus. Muo-production of exclusive vector mesons at high energies is well suited for this purpose. In this case the initial size of the vector meson is determined by the $\mathrm{Q}^{2}$, the virtuality of the photon. At a $\mathrm{Q}^{2}$ value of $1 \mathrm{GeV}^{2}$ the transverse size of the meson is roughly $2 / Q$, or about $0.4 \mathrm{fm}$, which is much smaller than the size of a normal hadron of about $1 \mathrm{fm}$. In the E665 kinematic region, an exclusively produced vector meson typically carries about $200 \mathrm{GeV}$. The corresponding formation length (the distance traveled by the emerged state before it grows into full size ) is roughly $2 \nu /\left(\mathrm{Q}^{2}+\mathrm{m}_{\mathrm{v}}^{2}\right)$ 
where $m_{v}$ is the mass of vector meson, about $50 \mathrm{fm}$, which is much larger than the size of even the heaviest nucleus. Therefore one expects the nucleus to be highly transparent to the vector mesons produced even at modest $Q^{2}$ values of 1 or $2 \mathrm{GeV}^{2}$.

The data used for this analysis were taken at the Fermilab Tevatron muon beam line with the E665 spectrometer. A detailed description of the spectrometer can be found else where. ${ }^{5}$ Here we will only briefly describe the components that are important to this analysis. The positive beam muon is momentum analysed with a beam spectrometer consisted of four stations of multi-wire proportional chambers and a dipole magnet. The mean beam energy was $470 \mathrm{GeV}$. The momentum resolution of the beam spectrometer is about $.5 \%$ at $500 \mathrm{GeV} / \mathrm{c}$. A remotely controlled target assembly cycles the targets every minute, thus greatly reduces the systematic effects associated with long term time variations of the running conditions. The scattered muon is identified as a track falling outside the projected beam region in the muon detector behind three meters of steel absorber. The momentum of the scattered muon and the final state hadrons are measured with an open geometry general purpose forward spectrometer consisted of two dipole magnets instrumented with multi-wire proportional and drift chambers. The spectrometer is augmented greatly by a fine grained electromagnetic calorimeter consisted of twenty planes of proportional tubes sandwiched with lead sheets one radiation length each. The momentum resolution of the forward spectrometer for the full length charged tracks is about $1 \%$ at $500 \mathrm{GeV} / \mathrm{c}$.

The variables employed to describe the exclusive vector meson production are defined as the following: $Q^{2}=-\left(p_{\mu}-p_{\mu}^{\prime}\right)^{2}, \nu=p . q / m_{p}, x_{b j}=Q^{2} / 2 m_{p} \nu, t=(q-v)^{2}$, $t^{\prime}=t-t_{\min }, z_{\mathrm{v}}=E_{\mathrm{v}} / \nu$, where $p_{\mu}, p_{\mu}^{\prime}, \mathrm{q}$ and $v$ are the four momenta of the incoming muon, the outgoing muon, the virtual photon and the vector meson respectively, $-Q^{2}$ is the invariant mass of the virtual photon, $\nu$ is the energy loss of the muon in the laboratory, $t$ is the the four momentum transfer squared between the vector meson and the nucleon, $t_{\min }$ is the minimum $\mathrm{t}$ allowed by the kinematics, and $z_{\mathrm{v}}$ is the ratio of the energy carried by the vector meson to the energy loss by the muon. In the following text $t$ will be used for $t^{\prime}$ without exception.

Events to be accepted by the present analysis were required to have two and only two oppositely charged hadrons present in the event in addition to the scattered muon ( the low energy recoil nucleon, with typical kinetic energy of less than .1 GeV, was not detected ). Inclusive and combinatorial contaminations were suppressed by requiring that $z_{\mathrm{v}}$ for the vector meson candidate be between 0.9 and 1.1 . Events from bremsstrahlung photon conversion were rejected using the calorimeter information by demanding that the energy and shower shape associated with the hadrons not be consistent with photons or electrons. To further reduce contributions from events in which additional particles were produced, but not reconstructed, the number of residual hits ( hits not used by the reconstructed tracks ) in the vertex drift chambers was limited to a value consistent with the normal level of spurious hits. An additional cut requiring the energy of both hadrons to be greater than $10 \mathrm{GeV}$ was imposed to reduce effects associated with less well-understood acceptance of the low-energy 
particles.

The invariant mass distributions obtained were first fitted to a p-wave BreitWigner form multiplied by a skewing factor $\left(\mathrm{m}_{\rho} / \mathrm{m}_{\pi \pi}\right)^{\mathrm{n}}$ assuming the hadrons are pions. The mass distributions for events that are not consistent with $\rho^{0}$ were then fitted to a form of a signal plus background assuming the hadrons are kaons. $M_{\rho}$ and $\mathrm{m}_{\phi}$ thus obtained agree well with the Particle Data Book values. $\Gamma_{\rho}$ and $\Gamma_{\phi}$ obtained are consistent with the Particle Data Book values smeared by the detector resolution.

Production of exclusive vector mesons from a nuclear target has two components: the coherent component corresponding to production off the nucleus as a whole and the incoherent component corresponding to production off the individual nucleons in the nucleus ( terms elastic and quasi-elastic for coherent and incoherent are also used in literature). The slopes of the $t$ distributions of a diffractive scattering process measure the physical sizes of the scatterer. Thus, for a nuclear target, one expects to see a $t$ distribution consisted of a region of steeply falling followed by a region falling with a much smaller slope. The observed $t$ distributions for hydrogen and lead targets are shown in Figs $1 \mathrm{a}$ and $1 \mathrm{~b}$, respectively. Regions corresponding to coherent and incoherent production are clearly identifiable in Fig. 1b.

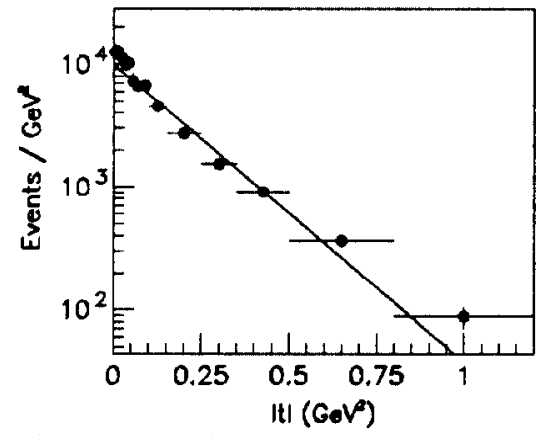

Fig. 1a $t$ distribution for $\rho$ and $\$$ condidates from o hydrogen torget. The curve is o fit to o*exp $(-b *|t|)$

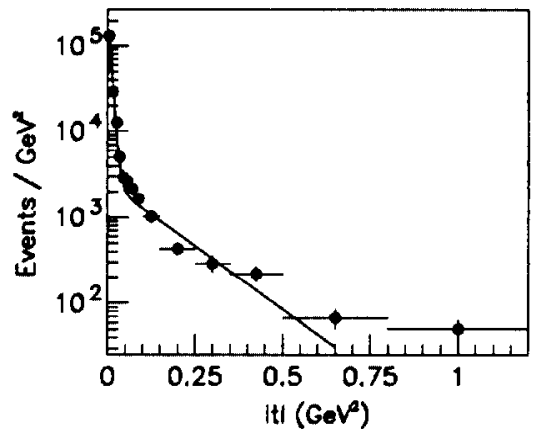

Fig. $10 t$ distribution for $p$ and $\uparrow$ condidates from o lead torget. The curve is a fit to a1.exp $(-b i=|t|)+a 2 \cdot \exp (-b 2 \cdot|t|)$

Separation of coherent and incoherent event samples are achieved by a cut in $t$. Events with $t$ values greater than $0.1 \mathrm{GeV}^{2}$ are treated as incoherent ( the same for all the targets ). Contributions from the coherent events were estimated by integrating the observed coherent exponential functions from $0.1 \mathrm{GeV}^{2}$ to infinity. The level of contaminations were less than $1 \%$ for all but the deuterium target for which the contamination is about $10 \%$. Contaminations of incoherent events to the coherent samples were subtracted statistically by extrapolating the incoherent distributions down to $t=0$ with a slope averaged over the observed incoherent slopes from all the targets (in doing so we assume that the $t$ slope associated with the nucleon production from different nucleus are identical ).

The ratios of the incoherent $\rho^{0}$ and $\phi$ production cross sections off deuterium, 
carbon, calcium and lead to that off hydrogen versus $Q^{2}$ are shown in Fig. 2a. The corresponding nuclear transparency $\mathrm{T}$, defined as $\sigma^{\mathrm{A}} / \mathrm{A} \sigma^{\mathrm{N}}$, as a function of $\mathrm{A}$, is plotted in Fig. $2 \mathrm{~b}$ for different $\mathrm{Q}^{2}$ regions. The ratios of coherent $\rho^{0}$ and $\phi$ production cross sections off calcium and lead to that off carbon versus $Q^{2}$ are shown in Fig. $3 \mathrm{a}$ and the corresponding transparency as a function of $\mathrm{A}$ is shown in Fig. $3 \mathrm{~b}$. The lines in Fig. $2 b$ are fits to $\mathrm{p}(1) * \mathrm{~A}^{\mathrm{p}(2)}$ and the lines in Fig. $3 \mathrm{~b}$ are fits to $(\mathrm{A} / 12)^{\mathrm{p}}$. It should be pointed out that experimentally the exclusive vector meson samples include contributions from production off nucleon excitation and resonance production ( to the extent that the $t$ is large enough to allow these processes to happen energetically ) in the case of light targets. However, the corresponding production mechanism does not exist in the heavy target cases. Therefore one would under-estimate the transparency if one were to normalize the cross sections to hydrogen or deuterium. Because of this we normalize the coherent production cross sections to carbon. It

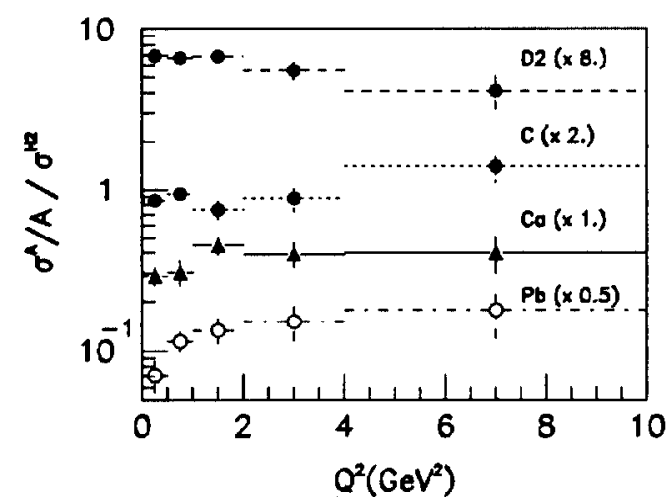

Fig. $20 \sigma / A / \sigma^{10}$ vs $Q^{2}$ for incoherent $\rho^{0} \& \&$ production. Note that the doto points hove been multiplied by $0.5,1.0,2.0$ and 8.0 respoctively for the four targets.

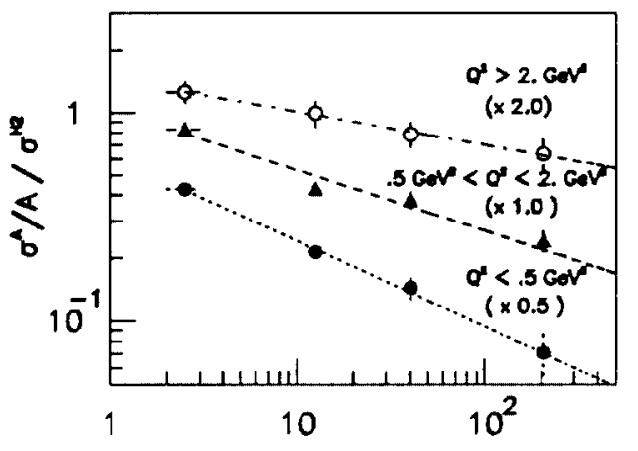

$A$

Fig. $2 b \quad \sigma^{A} / A / \sigma^{10}$ vs $A$ ot different $Q^{2}$ for incoherent $\rho^{*} \& \$$ production. The curves ore fits to $\sigma^{*} / A / \sigma^{\text {te }}=p(1) * A^{(x)}$. Note that the doto points hove been multiplied by 0.51 .0 and 2.0 respectively for the three $Q^{2}$ regions.

is seen that as the $\mathrm{Q}^{2}$ increases the per-nucleon cross sections from heavy targets all increase in both the coherent and incoherent cases ( the apparent drop in the deuterium to hydrogen ratio in Fig. $2 \mathrm{a}$ at the high $Q^{2}$ bins is being studied. Possible effects from the coherent contaminations have been studied by imposing a larger $t$ cut in selecting the incoherent sample. No significant changes was found. Other possible explanations for this drop include differences in the production mechanism between a proton and a neutron and nuclear characteristics). As $\mathrm{Q}^{2}$ increases the slopes of the $T$ versus $A$ lines in the coherent case increase and approach $1 / 3$ at high $Q^{2}$ as one would expect for a perfectly transparent nucleus ( derived from the fact that the scattering amplitude is proportional to $\mathrm{A}$, thus the differential cross section is proportional to $A^{2}$. After integrating over $\mathrm{t}$ one get a factor $b_{A}$ in the denominator which is proportional to $A^{2 / 3}$. Thus the production cross section integrated over $\mathrm{t}$, as what is presented here, would be proportional to $A^{4 / 3}$, leading to a $\mathrm{T}$ proportional 
to $\left.A^{1 / 3}\right)$. The lines in the incoherent case become less steep as $Q^{2}$ increases and the slopes of the lines approach 0 at high $Q^{2}$, implying a total production cross section proportional to $A$. This behavior in $T$ agrees well with what is expected from the color transparency arguments. As the $Q^{2}$ increases, the initial size of the produced mesons becomes smaller, and thus they experience a weaker color field on the way out. Therefore, the attenuation due to final-state interactions becomes smaller and more mesons produced in the nuclear interior emerge intact.

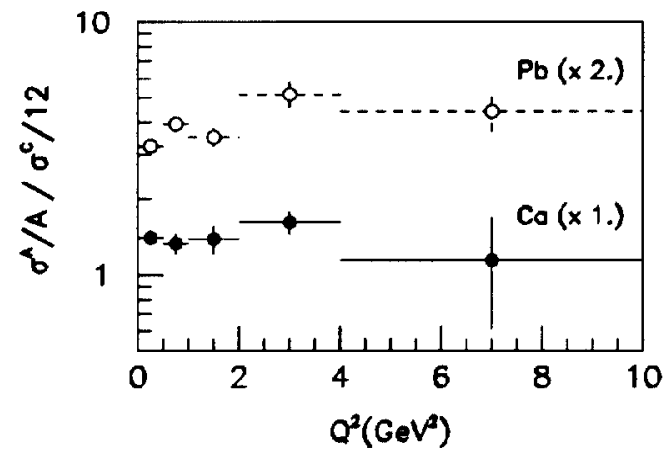

Fig. 3o $\sigma^{*} / A / \sigma^{\circ} / 12$ vs $\sigma^{2}$ for coherent $\rho^{0} \& \&$ praduction. Note that the doto points for leod hove been multiplied by 2.0 .

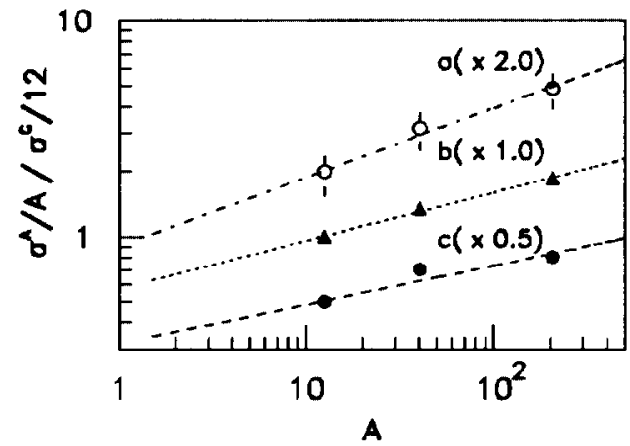

Fig. 3b $\sigma / A / \sigma^{*} / 12$ vs $A$ ot different $Q^{2}$ for coherent $\rho^{\circ} \&$ production. a) $Q^{2}>2 \mathrm{GeV}^{2}$. b) $0.5 \mathrm{GeV}^{2}<Q^{2}<2 \mathrm{GeV}^{2}$, c) $Q^{2}<0.5 \mathrm{GeV}^{2}$. The curves ore fits to $\sigma^{*} / A / \sigma^{\varepsilon} / 12=(A / 12)^{\prime}$. Note that the dato points hove been multiplied by $0.5,1.0$ and 2.0 respectively for the three $Q^{2}$ regions.

Effects due to the residual inclusive contaminations were estimated to be no more than $30 \%$ in the highest $\mathrm{Q}^{2}$ bin (where the effects were the largest). The uncertainty in $\mathrm{Q}^{2}$-independent relative normalization was estimated to be less than $7 \%$. These uncertainties should decrease with further studies. Effects due to secondary interactions and remaining photon conversion events were estimated to be small.

In summary we have measured the cross section ratios of incoherent exclusive $\rho^{0}$ and $\phi$ production off deuterium, carbon, calcium and lead to that off hydrogen and the cross section ratios of coherent exclusive $\rho^{0}$ and $\phi$ production off calcium and lead to that off carbon. Preliminary results show that the cross section ratios increase as the $Q^{2}$ increases, as suggested by the concept of color transparency.

\section{References}

1. T.H. Bauer et al, Rev. of Modern Phys. 50, 261 (1978).

2. S.J. Brodsky and A.H. Mueller, Phys. Lett. B206, 685 (1988).

3. L. Frankfurt and M. Strikman, Phys. Rep. 160, 235 (1988).

4. B.Z. Kopeliovich et al, Phys. Lett. B309, 179(1993).

5. M. Adams et al, Nucl. Inst. and Meth., A291, 533 (1990). 